J.-P. Paulet (dir.), 2000, Les très grandes villes dans le monde

Paris, CNED et SEDES, $240 \mathrm{p}$.

\title{
Bruno Valentin
}

\section{(2) OpenEdition}

\section{Journals}

Édition électronique

URL : http://journals.openedition.org/rge/2523

DOI : 10.4000/rge.2523

ISSN : 2108-6478

Éditeur

Association des géographes de l'Est

Édition imprimée

Date de publication : 1 janvier 2003

ISSN : 0035-3213

Référence électronique

Bruno Valentin, « J.-P. Paulet (dir.), 2000, Les très grandes villes dans le monde », Revue Géographique de I'Est [En ligne], vol. 43 / 1-2 | 2003, mis en ligne le 08 décembre 2010, consulté le 25 septembre 2020. URL : http://journals.openedition.org/rge/2523; DOI : https://doi.org/10.4000/rge.2523

Ce document a été généré automatiquement le 25 septembre 2020

Tous droits réservés 


\section{J.-P. Paulet (dir.), 2000, Les très grandes villes dans le monde}

Paris, CNED et SEDES, $240 \mathrm{p}$.

\section{Bruno Valentin}

\section{RÉFÉRENCE}

J.-P. Paulet (dir.), 2000, Les très grandes villes dans le monde, Paris, CNED et SEDES, 240 p.

1 Cet ouvrage propose aux lecteurs des réflexions sur l'explosion urbaine de la deuxième moitié du $\mathrm{XX}^{\mathrm{e}}$ siècle, avec une analyse sur la complexité des espaces agglomérés, sur les limites urbaines et les incidences sociales des grandes villes. Les aspects relatifs au fonctionnement et à la gestion durable de ces grandes entités urbaines aux formes imprécises sont également analysés.

2 Les éditions SEDES ont l'ambition de publier, chaque année, une synthèse sur les thèmes mis au programme des concours. Elle s'adresse donc aux étudiants qui préparent le CAPES et les Agrégations d'histoire et de géographie, mais également à tous les lecteurs intéressés par le thème traité. Cette introduction à la géographie des grandes villes est une synthèse sur les mutations géographiques des très grandes agglomérations dans le monde. L'analyse des rythmes et des facteurs de la croissance démographique des très grandes villes, complétée par celle des mécanismes et processus économiques, permettent de mieux comprendre l'organisation interne des grands organismes urbains et les difficultés de leur gestion. L'approche comparée des générations de grandes agglomérations s'appuie sur des exemples nombreux et variés.

3 Jean-Pierre Paulet s'efforce, en introduction et dans le chapitre premier, de faire un point épistémologique au sujet de la ville : il souligne la subjectivité et l'imprécision de la notion. À travers la profusion de termes, il retient la complexité de l'organisation, son rôle dans la globalisation de la société. On comprend rapidement que l'analyse morphologique ne peut suffire à expliquer cet «espace vécu», et qu'il faut donc 
compléter l'étude par des caractères fonctionnels. Il propose des mises au point sur les différents aspects du sujet, un état de la question intégrant des données à jour et des problématiques récentes. Une abondante illustration statistique, graphique et cartographique complète le texte.

Dans le monde, près de 150 agglomérations atteignent aujourd'hui 2 millions d'habitants. Cela pose le problème des méthodes et des concepts nécessaires pour décrire la généralisation planétaire des très grandes villes et les nouvelles formes de l'urbanisation. Cet ouvrage aborde la très grande ville sous les angles historique, démographique, spatial, fonctionnel, social, politique et culturel, en montrant les interactions entre ces différentes composantes. Mais il faut être attentif à prendre en compte les différentes échelles et l'approche globale ne doit pas faire oublier les singularités régionales ou locales. C'est pourquoi l'ouvrage présente aussi les villes de plus de deux millions d'habitants par grandes aires géographiques et comporte des analyses comparées et des études de cas.

5 Le chapitre 2 est le fruit du travail d'une équipe de spécialistes de géographie urbaine, alliant leurs compétences scientifiques de chercheurs, leurs expériences de terrain et leurs soucis pédagogiques d'enseignants. Ils nous entraînent dans le «cycle de vie urbain ", où l'urbanisation pousse à la concentration, puis à la suburbanisation et jusqu'à la désurbanisation. Les conséquences du mouvement, souvent négatives, conduisent à un mouvement de réurbanisation et de réhabilitation du centre («Gentrification»). L'étude s'appuie sur des exemples précis avec des villes nordaméricaines (edge cities, exurbs, gated communities et lone eagles). Le lecteur pourra apprécier la modélisation des cycles et l'accès aux définitions.

Dans le chapitre 3, Philippe Cadène nous guide dans une étude géohistorique des grandes villes des pays faiblement développés. L'étude des anciennes cités du Moyen Orient et de l'Afrique nord-saharienne montre que c'est le facteur politique qui joue un rôle prédominant dans la croissance urbaine.

7 Les chapitres 4,5 et 6 nous amènent à réfléchir sur l'agglomération dans le système monde. L'analyse permet de rendre compte d'évolutions similaires, selon des chronologies décalées. Mais elle souligne aussi la grande diversité des situations, qui tient à des spécificités historiques, politiques et culturelles, ainsi que les inégalités, qui relèvent de plus en plus de la place occupée par chaque ville dans la hiérarchie métropolitaine du système-monde. Ici, P. Cadène traite les dramatiques situations de certains quartiers pauvres, des problèmes d'accès au logement et de la paupérisation (Mexico et son bidonville Nezahualcoyotl). En bref, de la métropolisation à la bidonvilisation.

8 Enfin, dans les deux derniers chapitres, les auteurs traitent la théorie géographique de la métropolisation et la prospective pour une habitation durable des territoires. La compréhension des très grandes villes passe par une étude topochronique qui permet de mettre en exergue le rapport au temps inscrit dans les territoires. L'article a l'avantage de situer la géographie dans son champ d'investigation que représente le territoire urbain : l'auteur construit un référentiel entre l'habitant, le territoire, la vie collective (référentiel habitant, référentiel identité à une échelle régionale et macro-régionale). Le développement durable n'est pas en reste avec une analyse des grands dispositifs internationaux, dans lesquels l'auteur insiste sur le fait que le public doit être impliqué dans le processus de développement. 
9 L'ouvrage se termine par l'analyse de la logique touristique dans les grandes villes. Depuis 1950 on assiste à une sorte "d'urbanotropisme » particulièrement favorable aux grandes villes. Si la fréquentation est croissante, on note qu'elle est inégalement répartie. Ici la ville est perçue comme un mythe (du Golden Bridge à San Francisco au Parthénon d'Athènes), mais il est difficile d'établir un classement hiérarchique en fonction de l'attractivité patrimoniale des villes. La ville touristique est finalement « un espace transition» entre les bases du site urbain et le rôle postmoderne de la métropole.

10 L'ouvrage met en évidence avec beaucoup de clarté le rôle de la ville dans un ensemble global, la très grande ville posant la question du sacro-saint développement durable.

\section{AUTEUR}

\section{BRUNO VALENTIN}

Université Nancy 2 